\title{
Metodología de Diseño de Prendas Usando Textiles Impresos en 3D con Filamento PET Reciclado
}

\section{Garment Design Methodology Using 3D Printed Textiles with Recycled PET Filament}

\section{P. Restrepo-Salgado' ${ }^{1}$ D. Cortés-Sáenz ${ }^{1}$, S. E. Arbeláez-Ríos ${ }^{1}$}

1 Universidad Autónoma de Ciudad Juárez

\section{RESUMEN}

El plástico es el material más frecuentemente encontrado a escala global en costas y ambientes marinos. Asimismo, los residuos sólidos representan una fuente de emisión y reproducción de vectores, con aportaciones a la contaminación atmosférica en entornos urbanos. Incorporar materia prima derivada de desechos plásticos a la cadena de suministro para generar productos de alto valor, como lo sugiere el concepto de economía circular, ayudaría a disminuir la contaminación. Una forma de lograrlo es a través de la implementación de tecnologías en crecimiento como la manufactura aditiva (MA), con la cual se pueden manufacturar productos atractivos, innovadores y personalizables. La industria textil representa un nicho de oportunidad para la implementación de estos materiales y tecnologías, a partir de la generación de prendas con alto valor ecológico y emocional. Por lo tanto, se propone una metodología donde la fase 1 y 2 involucran la primera etapa del diseño guiado por materiales (MDD), la fase 3 habla sobre la definición del usuario y prenda a diseñar y, finalmente, la fase 4 y 5 se centran en el modelo de procesos representativos para el desarrollo actual de prendas de vestir. Se concluye que la fabricación de productos con alto valor ecológico, a partir de residuos plásticos, es una opción viable y económicamente eficiente siempre y cuando el producto se diseñe pensando en todas sus fases de ciclo de vida, se conozca el material, su comportamiento y cómo el usuario lo percibe.

PALABRAS CLAVE: textil impreso en 3D; economía circular; diseño de prendas; caracterización experiencial.

\section{ABSTRACT}

Plastic is the material most frequently found on a global scale in coasts and in the marine environment. Likewise, solid waste represents a source emission and vector reproduction, with contributions to air pollution in urban environments. Incorporating raw material derived from plastic waste into the supply chain to generate high-value products as suggested by the concept of circular economy, would help reduce pollution. One way to achieve this is through the implementation of growing technologies such as additive manufacturing (AM), with which attractive, innovative and customizable products can be manufactured. Therefore, a methodology is proposed where phase 1 and 2 involve the first stage of Material Driven Design (MDD), phase 3 talks about the definition of the user and garment to design and, finally, phases 4 and 5 are focus on the representative process model for today's clothes development. It is concluded that the manufacture of products with high ecological value, from plastic waste is a viable and economically efficient option as long as the product is designed with all its life cycle phases in mind, the material is known, its behavior and how the user perceives it.

KEYWORDS:3D printed textile; circular economy; garment design; experiential characterization.

Correspondencia:

DESTINATARIO: María Paulina Restrepo Salgado INSTITUCIÓN: Universidad Autónoma de Ciudad Juárez / Instituto de Ingeniería y Tecnología DIRECCIÓN: Av. del Charro 450 norte, col. Partido Romero, C. P. 32310. Ciudad Juárez, Chihuahua, México CORREO ELECTRÓNICO: mprestreposa92@gmail.com
Fecha de recepción: 30 de abril de 2021. Fecha de aceptación: 6 de octubre de 2021. Fecha de publicación: 29 de octubre de 2021.
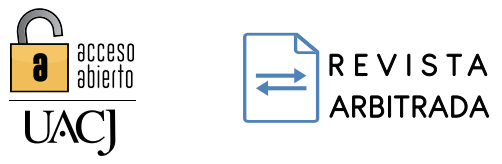


\section{INTRODUCCIÓN}

El diseño de productos debe cumplir con diversas etapas para cubrir diferentes requerimientos ${ }^{[1]}$. Estos aspectos abarcan el diseño visual, la manufactura del producto y las necesidades del mercado [2]. Además, se debe considerar la durabilidad, compatibilidad, modularidad o funciones multitarea del producto para minimizar el consumo de materia prima y mantenerlo circulando en bucles cerrados ${ }^{[3]}$. En relación a lo anterior, McCann ${ }^{[4]}$ menciona que para el diseño de prendas innovadoras se debe atender las necesidades del usuario final desde puntos de vista técnicos, funcionales, estéticos y culturales. Por lo tanto, presenta una herramienta que guía el proceso de investigación y desarrollo del diseño en la aplicación de tecnologías inteligentes en la creación de prendas de vestir.

Asimismo, Gill [5] habla sobre los enfoques para el desarrollo de prendas de vestir que incluyen pasos clave para definir las dimensiones básicas de las formas de patrones. Menciona que existen estos tres enfoques de producción de prendas que dependen del enfoque de producción que se le desee dar al diseño y desarrollo de este producto: 1) listo para usar (RTW: Ready to Wear), 2) hecho a la medida (bespoke) y 3) personalización en masa. Por su parte, Karana [6] presenta la metodología de diseño guiado por materiales (MDD: Material Driven Design), la cual permite diseñar para las experiencias con y para un material. Como parte de esta metodología, desarrolló una herramienta llamada caracterización experiencial, la cual evalúa cuatro dimensiones que permiten investigar cómo se percibe o se acepta un material, es decir, qué hace que las personas piensen, sientan y hagan, lo cual es esencial cuando los materiales se toman como punto de partida del proceso creativo y se exploran por su potencial para evocar una experiencia de producto única y significativa ${ }^{[7]}$.

Herrmann ${ }^{[8]}$ menciona que los textiles y la ropa son parte fundamental de la vida cotidiana y elemento muy importante de la economía. Sin embargo, el fenómeno de fast fashion trabaja de forma lineal (Figura 1), haciendo que la vida útil de una prenda sea más corta. Por tal razón, sugiere transformar la manera en que la ropa es diseñada, vendida y usada (Figura 2). En consecuencia, la pieza diseñada también debería estar pensada para su futuro reciclaje, puesto que este aspecto puede hacer que crezca positivamente la cantidad de material apto para ser reciclado y recuperado [9]. Por lo tanto, implementar el diseño circular en los procesos ayuda a contrarrestar la obsolescencia y a mantener al producto lo más cerca de su propósito original. También, permite que productos usados se conviertan en un elemento integral de la materia prima ${ }^{[10]}$.

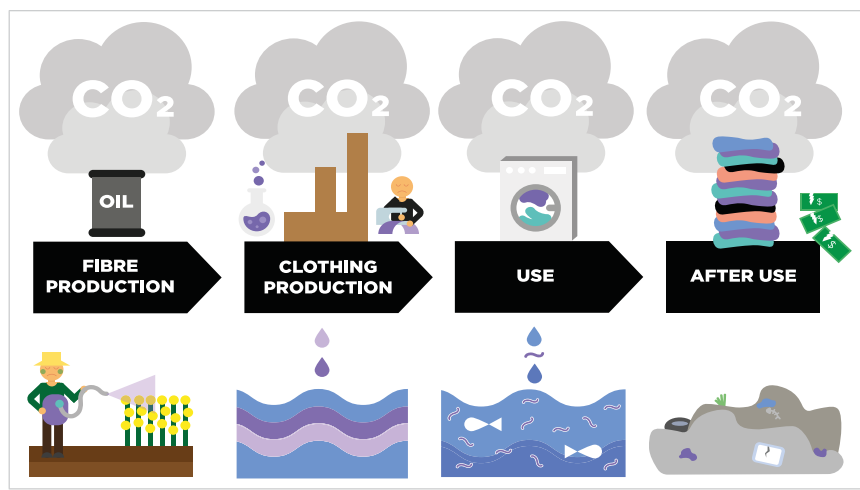

Figura 1. Ciclo de vida actual de una prenda de vestir, proceso lineal. Reproducida de ${ }^{[8]}$.

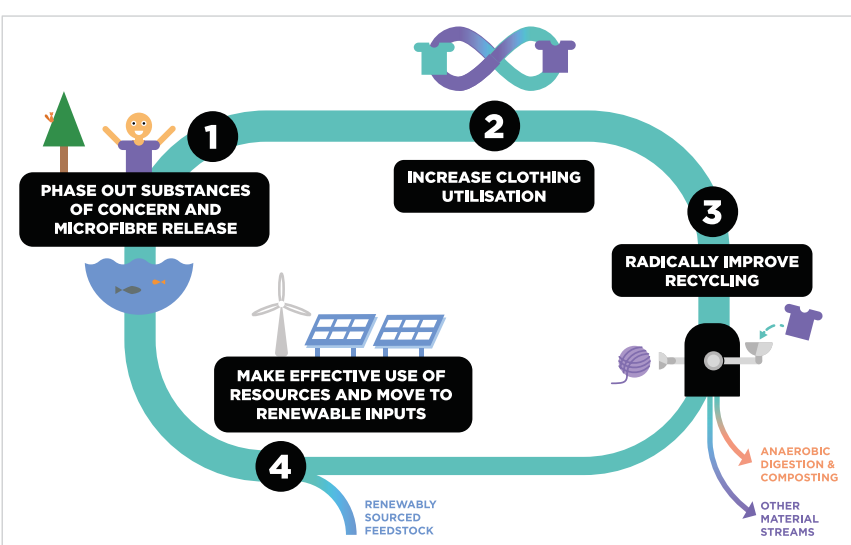

Figura 2. Modelo circular para la economía textil. Reproducida de ${ }^{[8]}$.

Adoptar y ajustar tecnologías innovadoras al sector de la confección puede generar productos más atractivos. En este caso, la MA permitirá manufacturar textiles más limpios y sostenibles [11]. Diversas tecnologías de MA han sido desarrolladas hasta el momento y, según Wong [12], estas pueden clasificarse de acuerdo a la presentación del material que usen, sean polvos, líquidos o sólidos. Por ejemplo, la tecnología de modelado por deposición fundida (FDM: Fused Deposition Modeling) usa como materia prima diversos plásticos, mayormente en forma de filamento. Por su parte, FormFutura ${ }^{[13]}$ ha desarrollado filamento a partir de polietileno de tereftalato reciclado ( $\mathrm{rPET}$ ), en combinación con otros agentes.

De acuerdo con Aizenshtein [14], todos los desechos de polímeros, incluido el tereftalato de polietileno (PET: polyethylene terephthalate), comprenden más del $50 \%$ de 
los desechos sólidos provenientes de hogares (SHW: solid household wastes), cifra consecuente con el crecimiento del rol y la importancia del plástico debido al aumento poblacional junto con sus efectos. En busca de mitigar la contaminación por plástico, en 2014, aproximadamente el $71 \%$ de los paquetes de PET recolectados en el mundo se procesaron en fibra. En este sentido, Zhang ${ }^{[15]}$ menciona que convertir una gran cantidad de desechos de botellas PET en productos textiles de alto valor es considerada una alternativa ecológica y económicamente eficiente con grandes beneficios ambientales

En relación a lo anteriormente expuesto, Grain [16] realizó un estudio cuyo objetivo era examinar posibilidades para el diseño y fabricación de prendas de moda impresas en 3D, utilizando filamentos rPET y así poder determinar si era factible utilizar este material para crear piezas de moda portátiles o escultóricas, a través del diseño, desarrollo, muestreo y pruebas, mientras se utilizaba un material totalmente sostenible.

Asimismo, algunos diseñadores se han atrevido a utilizar las tecnologías de AM para producir sus prendas de vestir. La diseñadora Gabriela Ligenza realizó un sombrero impreso en nylon mediante la tecnología sinterizado selectivo por láser (SLS: selective laser sintering). Esta diseñadora ha mostrado la variedad y el potencial que tienen las tecnologías de impresión 3D dentro del diseño y fabricación de accesorios contemporáneos [17]. De igual forma, la diseñadora Iris Van Herpen ha realizado proyectos con diferentes tecnologías de manufactura aditiva, por ejemplo, su colección Crystallization y Escapism en las cuales utilizó sinterizado láser selectivo, su colección Voltage fue impresa en la tecnología Polyjet [18]. Por su parte, Kim [19] realizó una investigación para estudiar la implementación y mejora de la tecnología FDM (fused deposition modeling) en la industria de la moda. Como material de impresión utilizaron filamento de poliuretano termoplástico (TPU: thermoplastic polyurethane) y acrilonitrilo butadieno estireno (ABS: acrylonitrile butadiene styrene).

Kirsten Lussenburg [20] aplica el MDD para explorar cómo esta metodología podría ser usada en un proyecto donde la impresión 3D es el método de manufactura principal. En este estudio se utilizó como material de impresión el ácido poliláctico (PLA: polylactic acid) y la tecnología FDM. Por otra parte, dice que los textiles impresos en 3D (3DP Textiles) (Figura 3) son materiales semidesarrollados, una combinación entre material, es- tructura y proceso (MSP), que son aspectos que definen los dominios y oportunidades. De acuerdo con la investigadora, el requerimiento principal es la flexibilidad.

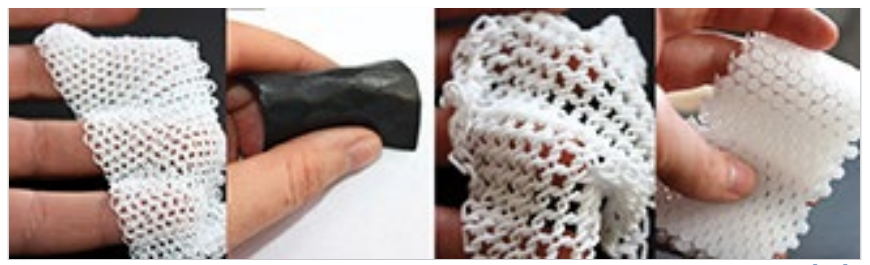

Figura 3. Ejemplo de textiles impresos en 3D. Reproducida de ${ }^{[20]}$.

En definitiva, es necesario generar propuestas que posibiliten recuperar e incorporar los desechos de plásticos a la cadena suministro de forma tal que el valor del producto se mantenga en el tiempo. Este documento tiene como objetivo presentar una propuesta metodológica que permita generar prendas de vestir a partir de textiles impresos por medio de la tecnología FDM fabricados a base de PET reciclado, haciendo uso de diferentes métodos y herramientas de diseño.

\section{MÉTODO}

Para generar la propuesta metodológica, se partió de una investigación de necesidades que permitiera determinar un nicho de oportunidad para el desarrollo de productos sostenibles.

Se realizó una búsqueda de antecedentes y una revisión teórica en bases de datos como ScienceDirect, Springer y Scopus, y en buscadores como Google Scholar. Se revisaron antecedentes (Figura 4) relacionados con el diseño de productos, impresión $3 \mathrm{D}$, filamento $\mathrm{PET}$ reciclado, economía circular y contaminación ambiental. A partir de esta búsqueda se encontró un nicho de oportunidad en el diseño y desarrollo de accesorios y prendas con ayuda de la tecnología (FDM).

Por otra parte, la revisión teórica (Figura 5) se centró en tres temas principales: materiales, diseño y desarrollo de productos y sostenibilidad, permitiendo identificar métodos y herramientas que se ajustarán al nicho de oportunidad anteriormente establecido.

A partir de estos hallazgos, se realizó la propuesta metodológica que se presenta a continuación, la cual expone cada uno de los métodos y herramientas que se sugieren ejecutar para diseñar una prenda de vestir a partir de filamento rPET fabricada con la tecnología FDM. 


\begin{tabular}{|l|l|l|}
\hline $\begin{array}{l}\text { Revisión de } \\
\text { antecedentes }\end{array}$ & $\begin{array}{l}\text { El consumo y desecho de plásticos es una } \\
\text { problemática mundial. Uno de estos materiales es } \\
\text { el tereftalato de polietileno (PET), que es } \\
\text { considerado uno de los grandes contaminantes. }\end{array}$ & $\begin{array}{l}\text { Se realizó una búsqueda de ideas acerca de cómo } \\
\text { generar artículos de valor a través del PET reciclado } \\
\text { (rPET). Se encontró un nicho de oportunidad en la } \\
\text { industria textil y de la moda a partir de la tecnología } \\
\text { de manufactura aditiva FDM. }\end{array}$ \\
$\begin{array}{l}\text { Existe un nuevo modelo económico llamado } \\
\text { economía circular, que pretende generar } \\
\text { circularidad en cada una de las etapas del ciclo de } \\
\text { vida de un producto y no dejar que se pierda el } \\
\text { valor que tiene. }\end{array}$ & $\begin{array}{l}\text { Cómo la economía circular está relacionando los } \\
\text { aspectos anteriores, desechos, textiles, moda y } \\
\text { nuevas tecnologías. }\end{array}$ \\
\hline
\end{tabular}

Figura 4. Resumen de revisión de antecedentes.

\begin{tabular}{|c|c|c|}
\hline \multirow{4}{*}{$\begin{array}{l}\text { Marco } \\
\text { teórico }\end{array}$} & \multirow{2}{*}{$\begin{array}{l}\text { Materiales: } \\
\text { - Características de los } \\
\text { plásticos. } \\
\text { - Características del PET y } \\
\text { del rPET. } \\
\text { - Manufactura Aditiva. } \\
\text { - Tecnología FDM. }\end{array}$} & \multirow[b]{2}{*}{$\begin{array}{l}\text { Selección del material y provee- } \\
\text { dor: Reform rPET fabricado por } \\
\text { FormFutura. }\end{array}$} \\
\hline & & \\
\hline & $\begin{array}{l}\text { Diseño y desarrollo de } \\
\text { producto: } \\
\text { - Diseño de producto. } \\
\text { - Metodología Diseño. } \\
\text { Guiado por Materiales } \\
\text { (MDD). } \\
\text { - Diseño circular - diseño } \\
\text { para la economía circular. } \\
\text { - Diseño de vestuario. } \\
\text { - Textiles impresos en 3D. }\end{array}$ & $\begin{array}{l}\text { - Etapas del dise.ño y desarrollo } \\
\text { de producto. } \\
\text { - Caracterización experiencial } \\
\text { - Se identifican estrategias del } \\
\text { diseño circular y se seleccionan } \\
\text { tres de ellas. } \\
\text { - Identificación del usuario. } \\
\text { - Patrones de textiles impresos en } \\
\text { 3D. }\end{array}$ \\
\hline & $\begin{array}{l}\text { Sostenibilidad: } \\
\text { - Métodos que soportan el } \\
\text { ecodiseño. } \\
\text { - Métodos que soportan la } \\
\text { economía circular. } \\
\text { - Nueva economía textil } \\
\text { basada en la economía } \\
\text { circular. } \\
\text { - Objetivos y metas del } \\
\text { desarrollo sostenible de la } \\
\text { ONU. }\end{array}$ & $\begin{array}{l}\text { - Diseño para } x \text {, método común } \\
\text { que soporta el ecodiseño y la } \\
\text { economía circular. También } \\
\text { ayuda a soportar alguna de las } \\
\text { estrategias de la economía } \\
\text { circular. } \\
\text { - La nueva economía textil ayuda } \\
\text { a visualizar requerimientos del } \\
\text { producto. } \\
\text { - Cumplir las metas } 9.4,12.4 \text { y } \\
12.5 \text { de los objetivos del desa- } \\
\text { rrollo sostenible de la ONU. }\end{array}$ \\
\hline
\end{tabular}

Figura 5. Revisión teórica.

\section{RESULTADOS}

La metodología propuesta como resultado del presente trabajo incluye cinco fases, de las cuales las primeras dos se realizan con ayuda de la primera etapa del MDD propuesto por Karana [6], que se enfoca en conocer el material desde características técnicas y experienciales. Kirsten Lussenburg ${ }^{[20]}$ habla detalladamente sobre cómo conocer y entender un textil impreso desde la perspectiva del MDD.

\section{FASE 1: Selección del textil impreso en 3D}

Se sugiere hacer una búsqueda en las librerías web que se muestran en la Figura 6, las cuales contienen gran variedad de modelos 3D. La búsqueda se debe limitar por los siguientes requerimientos: estructura fina y de múl- tiples ensambles, geometría pequeña con aristas poco pronunciadas, preferiblemente curvas [20].

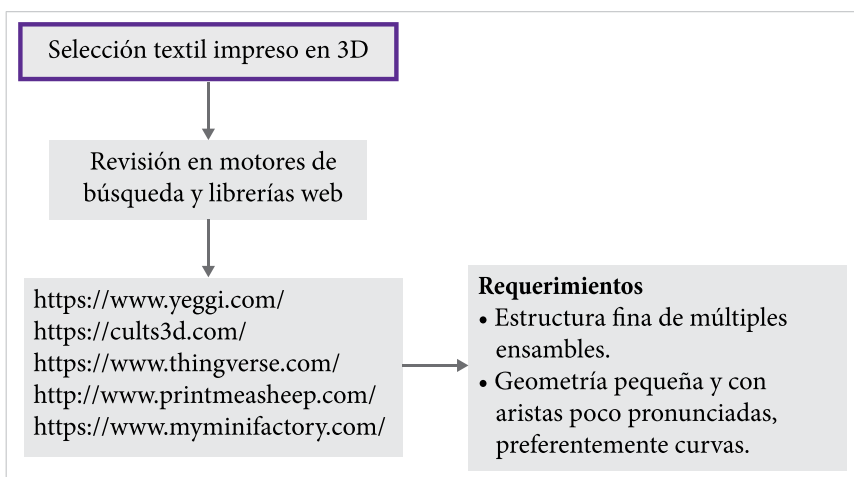

Figura 6. Revisión en motores de búsqueda y librerías web.

\section{FASE 2: Impresión y evaluación de los textiles impresos en 3D}

Selección del material de impresión. Se sugiere revisar en el mercado los diferentes proveedores de filamento rPET. En este caso se utiliza filamento de Reform rPET [13]. A partir de la ficha técnica entregada por el fabricante, se establecen los parámetros de impresión, tales como temperatura de impresión, temperatura de cama y velocidad del ventilador. Asimismo, se debe hacer una comparativa entre las propiedades mecánicas del rPET y el PET.

Caracterización del proceso de impresión. Como se muestra en la Figura 7, se sugiere usar el software Ultimaker Cura para identificar y modificar parámetros de impresión, tales como espesor de capa, densidad de relleno, temperatura de impresión, temperatura de cama, velocidad de impresión, velocidad del ventilador, generación de soporte. Esta herramienta computacional provee información como el tiempo de fabricación y cantidad de material utilizado. Después, estos parámetros e información se organizan en tablas para cada uno de los textiles impreso seleccionados. Una vez que 
se ajustan los parámetros de impresión, se realiza la impresión de los modelos de textiles haciendo uso de una impresora 3D. En este caso, la impresora es marca Creality modelo Ender 3 de tecnología FDM. Además, se propone observar el comportamiento del material durante la impresión y posterior a ella para determinar si se deben ajustar los parámetros de impresión.

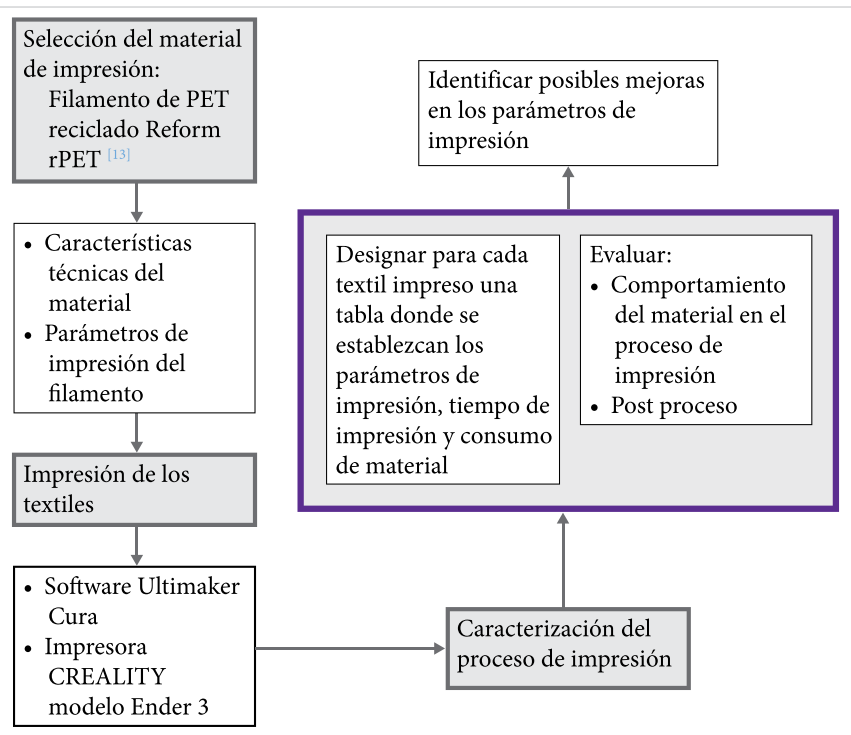

Figura 7. Caracterización del proceso de impresión.

Evaluación del textil. Como se observa en la Figura 8, esta evaluación se basa en dos encuestas, una donde se evalúan aspectos geométricos, de tamaño y función, y la otra evalúa características experienciales.

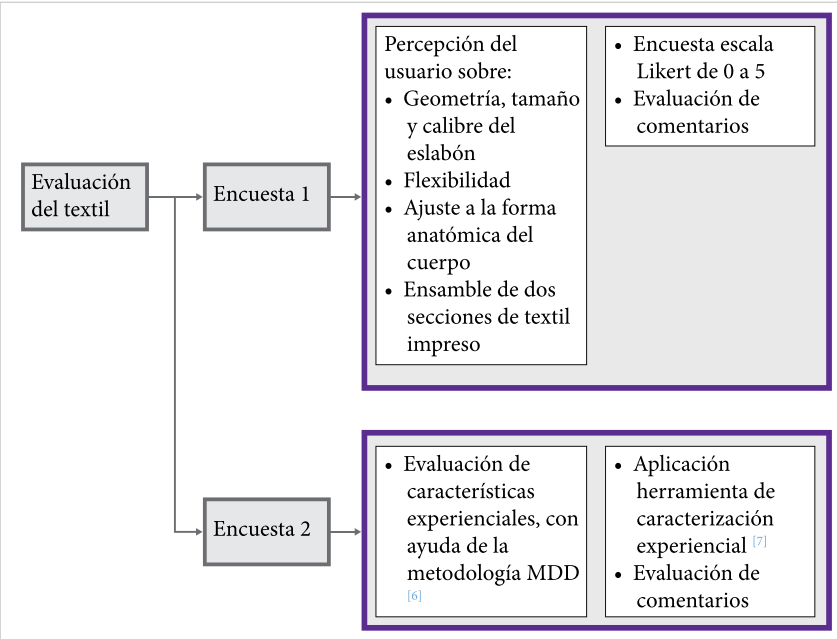

Figura 8. Evaluación textil.

Encuesta 1. En esta se utiliza la escala Likert para evaluar la percepción del usuario sobre la geometría, tamaño y calibre del eslabón (piezas que conforman el textil impreso) (Figura 9). También, se pregunta sobre la flexibilidad del material (es decir, si caía libremente), si este se ajusta a la forma anatómica del cuerpo. Finalmente, se pide ensamblar dos secciones de malla y calificar el nivel de dificultad de la tarea (encuesta propuesta por los autores).

¿Qué tanto le agrada la geometría del eslabón?

Marca solo un óvalo

\begin{tabular}{ccccccc}
0 & 1 & 2 & 3 & 4 & 5 \\
\hline Poco $\square$ & $\square$ & & $\square$ & $\square$ & $\square$ & $\square$ Mucho \\
\hline
\end{tabular}

¿Qué tanto le agrada el tamaño del eslabón?

Marca solo un óvalo

\begin{tabular}{ccccccc}
0 & 1 & 2 & 3 & 4 & 5 \\
\hline Poco $\square$ & $\square$ & $\square$ & $\square$ & $\square$ & $\square$ Mucho
\end{tabular}

$¿$ Cree que el textil es flexible (es decir, que cae libremente, se dobla) de acuerdo a los eslabones que lo conforman?

Marca solo un óvalo

\begin{tabular}{ccccccc}
0 & 1 & 2 & 3 & 4 & 5 \\
\hline Poco $\square$ & $\square$ & $\square$ & & \\
\hline
\end{tabular}

¿Le parece adecuado el calibre (grosor) del eslabón?

Marca solo un óvalo

\begin{tabular}{ccccccc}
0 & 1 & 2 & 3 & 4 & 5 \\
\hline Poco $\square$ & $\square$ & & $\square$ & $\square$ & $\square$ & \\
\hline
\end{tabular}

¿Cómo percibió la tarea de ensamblar dos secciones del textil impreso?

Marca solo un óvalo

$\begin{array}{ccccccc}0 & 1 & 2 & 3 & 4 & 5 \\ \text { Poco } \square & \square & \square & \square & \square & \square \text { Mucho }\end{array}$

¿Cree que el textil impreso se ajusta a la forma anatómica del cuerpo?

Marca solo un óvalo

\begin{tabular}{cccccc}
0 & 1 & 2 & 3 & 4 & 5 \\
\hline Poco $\square$ & $\square$ & & & $\square$ & $\square$
\end{tabular}

Figura 9. Encuesta de forma y función.

Encuesta 2. Se aplica la herramienta propuesta por Camera y Karan ${ }^{[7]}$ para la caracterización experiencial (Figura 10). Ahí se evalúan cuatro dimensiones: performativa, sensorial, afectiva e interpretativa. Esta encuesta es posible adaptarla a Google forms. De acuerdo con Kirsten Lussenburg [20], se aplica de forma presencial y a un grupo igual o mayor a 10 personas. 

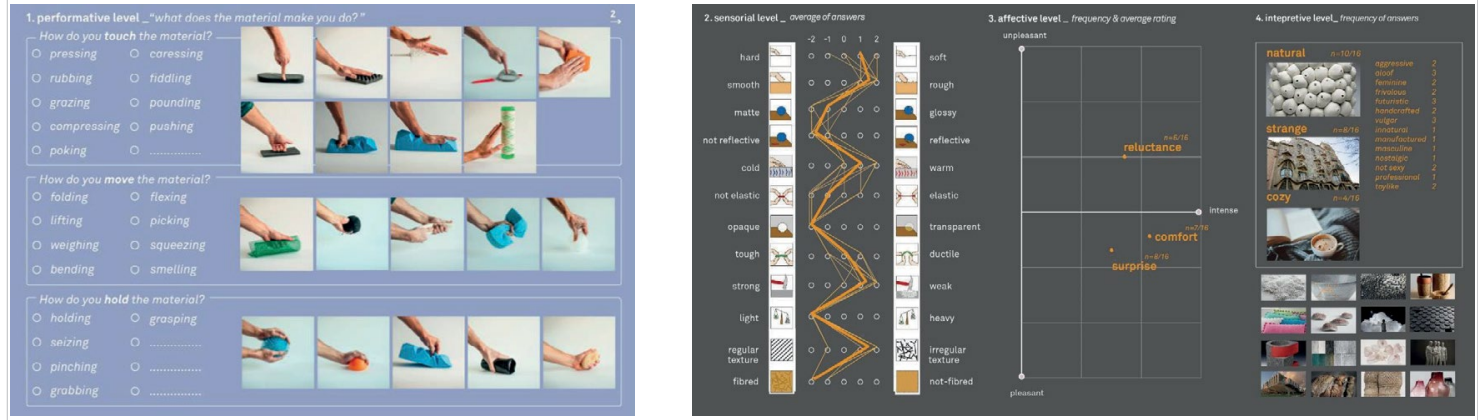

Figura 10. Herramienta de caracterización experiencial Ma2E4. La primera fotografía muestra el vocabulario performativo y la segunda es un ejemplo de cómo analiza y representa los datos recabados. Reproducida de [7].

Definición del usuario. Siguiendo con la etapa conceptual, se procede a identificar al usuario final a partir de lo sugerido por Gill [5]. El primer paso es definir las características que debe tener el usuario. Además, se deben tener en cuenta algunos requerimientos, como la demanda del cuerpo, demanda de la actividad, que el producto sea adecuado para el propósito y finalmente consideraciones estéticas y funcionales. Una vez se precisan estas características, se contrastan con perfiles de consumidor ya determinados, como los propuestos por Bell [21].

Definir la prenda a diseñar. Se concretan las siguientes necesidades para la prenda: diseño atemporal, diseño para la durabilidad del producto, diseño para fácil mantenimiento y reparación, diseño para el fácil desensamble. En busca de dar respuesta a estas necesidades, se propone realizar una entrevista a expertos para realizar un benchmarking y establecer conceptos de posibles prendas a confeccionar. Posteriormente, aplicar una matriz Pugh para seleccionar el concepto que mejor se adapte a los requerimientos anteriormente establecidos a partir de las encuestas y en las entrevistas a expertos. Lo anterior se ve resumido en la Figura 11.

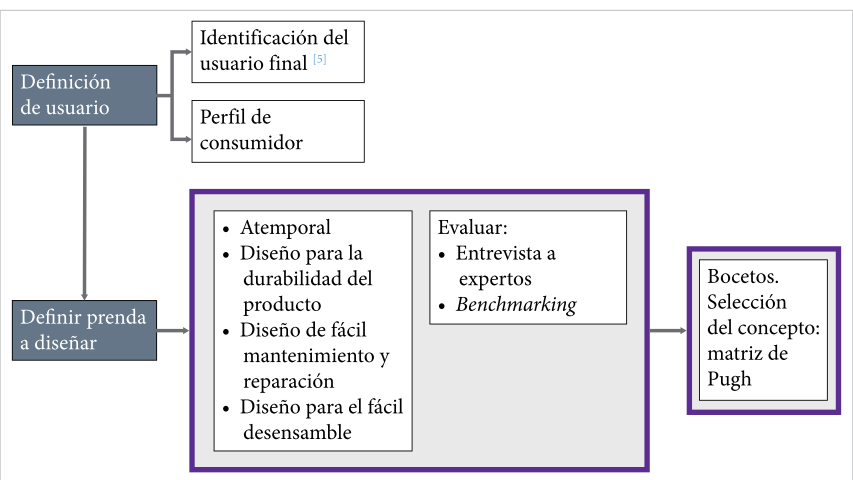

Figura 11. Definición de usuario y prenda a diseñar.
A partir de la fase 4, se empieza a trabajar sobre el modelo de procesos representativos para el desarrollo actual de prendas de vestir (Figura 12), haciendo uso del enfoque hecho a la medida (bespoke) ${ }^{[5]}$. En esta fase se trabajará con la parte de medición y construcción del patronaje.

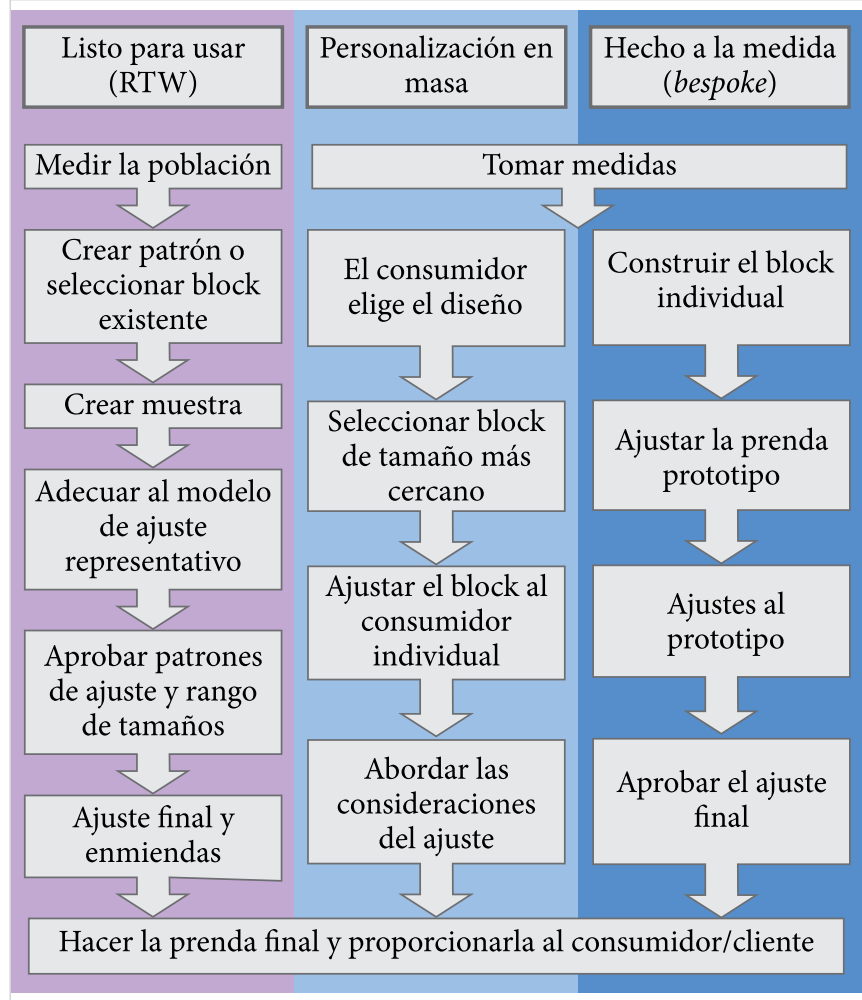

Figura 12. Procesos representativos para el desarrollo actual de prendas de vestir. Traducida de [5].

Tomar medidas y crear el patronaje de la prenda. Se toman las medidas del cliente con ayuda de una cinta métrica y a partir de ellas se genera el patronaje de la prenda. Se aconseja utilizar la herramienta computacio- 
nal Marvellous Designer, que permite trabajar con textiles de forma realista, realizar los patrones para confeccionar productos, importar texturas y finalmente unirla para generar un modelo 3D del producto.

\section{FASE 5: Desarrollo}

Prototipado. Se imprimen segmentos del textil de acuerdo a la capacidad de la impresora, luego se unen de acuerdo al patronaje de la prenda establecida.

Validación del prototipo. Continuando con el enfoque Hecho a la medida, se realiza una validación del prototipo (Figura 13) para ajustarlo mejor a las necesidades y gustos del usuario, así como para conocer aspectos técnicos como durabilidad técnica y huella ecológica, estimado de cuánto material se recupera por prenda.

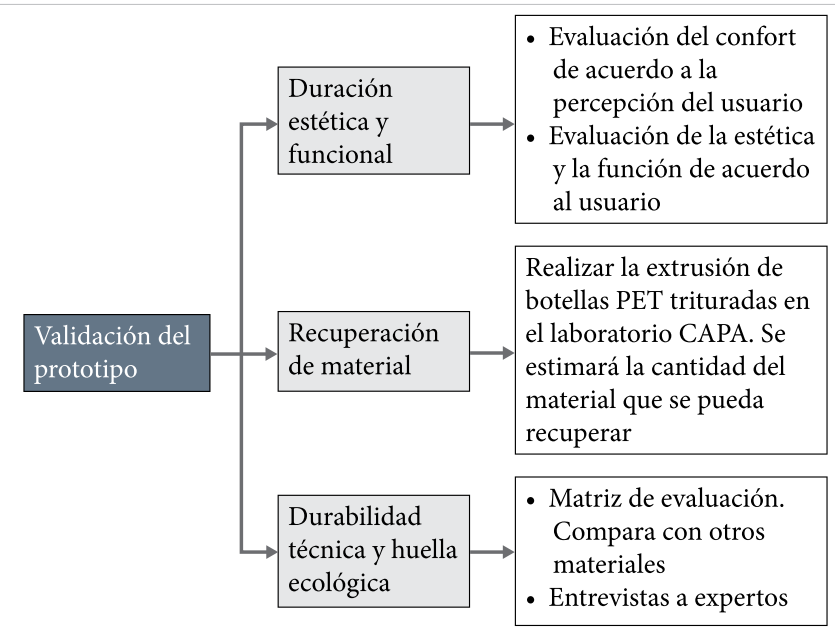

Figura 13. Validación del prototipo.

Ajustes al prototipo y aprobación del prototipo final. La validación y evaluación del primer prototipo permite realizar mejoras para el producto final. En esta etapa, el equipo de diseño tendrá que tomar decisiones para implementar dichas mejoras y así aprobar el modelo final.

\section{Confeccionar la prenda final y proveer al consumi-} dor. En este punto, la prenda ya estará lista para ser entregada al usuario. Sin embargo, no solo se trata de proveer un producto sino también de ofrecer un servicio con el fin de aumentar la esperanza de vida de dicho elemento. Estas estrategias de servicios pueden ser servicio de reparación y disposición de material.

\section{CONCLUSIONES}

La recuperación de residuos plásticos cada vez toma mayor importancia, pues los niveles de contaminación por estos elementos han tenido un incremento considerable en las últimas décadas. Por tanto, opciones como la de fabricar productos con alto valor ecológico a partir de residuos plásticos son viables y económicamente eficientes.

Un producto debe ser diseñado pensando en todas sus fases de ciclo de vida, puesto que se puede reducir el uso de materia prima, se pueden identificar técnicas de manufactura más eficientes y mejorar funcionalidad del mismo. Asimismo, se puede aumentar la probabilidad de que sea reusado, reparado o reciclado en un futuro.

Conocer el material, su comportamiento y cómo el usuario lo percibe permite diseñar adecuadamente y tomar decisiones acertadas respecto a la experiencia que se desea transmitir a través del material. En este sentido, el valor emocional posibilita que el producto tenga una esperanza de vida más amplia.

De acuerdo a lo anterior, la implementación de la tecnología FDM para le creación de prendas posibilita el uso de un solo material para la creación del textil y de las uniones. También, al ser una tecnología en crecimiento que ofrece la fabricación de elementos personalizados, genera expectativa y fascinación en el usuario. Además, permite disminuir el consumo de material puesto que los desechos son mínimos. Aun así, estos desechos exiguos se pueden disponer de forma correcta para generar nuevos filamentos.

\section{REFERENCIAS}

[1] K. T. Ulrich y S. D. Eppinger, Diseño y desarrollo de productos. McGraw-Hill, 2003, 409 pp.

[2] M. Baxter, Product design. Practical methods for the systematic development of new products, $1 .{ }^{a}$ ed. CRC Press, 1995, $304 \mathrm{pp}$.

[3] K. Medkova y B. Fifield, "Circular Design - Design for Circular Economy", en Lahti Cleantech Annual Review 2016, K. Cura, ed., Finlandia: Lahti University of Applied Sciences, 2016, pp. 32-47. 
[4] J. McCann, R. Hurford y A. Martin, "A design process for the development of innovative smart clothing that addresses end-user needs from technical, functional, aesthetic and cultural view points", Ninth IEEE International Symposium on Wearable Computers (ISWC'05), 2005, pp. 70-77, doi: 10.1109/ISWC.2005.3.

[5] S. Gill, "A review of research and innovation in garment sizing, prototyping and fitting", Text. Prog., vol. 47, no. 1, pp. 1-85, 2015, doi: 10.1080/00405167.2015.1023512.

[6] E. Karana, B. Barati, V. Rognoli y A. Zeeuw van der Laan, "Material Driven Design (MDD): A Method to Design for Material Experiences", Int. J. Des., vol. 9, no. 2, pp. 35-54, 2015. [En línea]. Disponible en: www. ijdesign.org/index.php/IJDesign/article/view/1965

[7] S. Camera y E. Karana, "Experiential Characterization of Materials: toward a toolkit", DRS2018 Catal., vol. 4, no. ag., 2018, doi: 10.21606/drs.2018.508.

[8] Ellen MacArthur Foundation, "A new textiles economy: Redesigning fashion's future”, Circular Fibres Initiative, 2017. [En línea]. Disponible en: static1.squarespace. com/static/5afae80b7c93276139def3ec/t/5b07ea5f8 8251b7468549158/1527245413992/A-New-TextilesEconomy_Full-Report_Updated_1-12-17.pdf

[9] M. K. Eriksen y T. F. Astrup, "Characterisation of source-separated, rigid plastic waste and evaluation of recycling initiatives: Effects of product design and source-separation system," Waste Manag., vol. 87, pp. 161-172, 2019, doi: 10.1016/j.wasman.2019.02.006.

[10] Y. E. Elmogahzy, "Engineering design for sustainability in the textile and garment industry", en Engineering Textiles. Integrating the Design and Manufacture of Textile Products, Y. E. Elmogahzy, ed. Woodhead Publishing, 2020, cap. 5, pp. 85-117.

[11] K. Chatterjee y T. K. Ghosh, "3D Printing of Textiles: Potential Roadmap to Printing with Fibers", $A d v$. Mater., vol. 32, no. 4, pp. 1-24, 2020, doi: 10.1002/ adma.201902086.

[12] K. V. Wong y A. Hernandez, "A Review of Additive Manufacturing," ISRN Mech. Eng., 2012, doi: $10.5402 / 2012 / 208760$.
[13] Formfutura, "ReForm rPET - Off-Black | Formfutura", Formfutura.com. Disponible en: www.formfutura.com/ shop/product/reform-rpet-off-black-974 (consulta: sept. $5,2020)$.

[14] E. M. Aizenshtein, "Bottle Wastes - to Textile Yarns", Fibre Chem., vol. 47, no. 5, pp. 343-347, 2016, doi: 10.1007/ s10692-016-9691-8.

[15] R. Zhang et al., "PET bottles recycling in China: An LCA coupled with LCC case study of blanket production made of waste PET bottles", J. Environ. Manage., vol. 260, 2020, doi: 10.1016/j.jenvman.2019.110062.

[16] E. Grain, "3D Printing Fashion With Recycled Polyester: A Sustainable Journey", presentada en la IFFTI - BIFT 2016 International Conference, Beijing, feb. 18, 2016, no. 2015.

[17] S. Holmes, "Millinery marvel - poem transformed into hat for Royal Ascot using 3D printing", Develop3d.com. develop3d.com/develop3d-blog/millinery-marvelpoem-transformed-into-hat-for-royal-ascot-using-3dprintin/ (consulta: mar. 9, 2021).

[18] E. Grain, "A Design-driven approach to 3D printing textiles from recycled polyester", presentada en la International Biennial Making Futures Research Conference: Crafting a Sustainable Modernity - Towards a Maker Aesthetics of Production and Consumption, Plymouth, Reino Unido, sept. 21, 2017, no. V.

[19] S. Kim, H. Seong, Y. Her y J. Chun, "A study of the development and improvement of fashion products using a FDM type 3D printer", Fash. Text., vol. 6, no. 9, 2019, doi: 10.1186/s40691-018-0162-0.

[20] K. Lussenburg, N. van der Velden, Z. Doubrovski, J. Geraedts y E. Karana, "Designing with 3D Printed Textiles: A case study of Material Driven Design", en $i C A T$ 2014: Proceedings of the 5th International Conference on Additive Technologies, Viena, Austria, oct. 16, 2014.

[21] A. Bell, "2022 El Consumidor del Futuro". WGSN Insight, p. 14, 2020. 